\title{
Autoantibodies and liver disease: Uses and abuses
}

\author{
Marilyn V Zeman MD MSc FRCPC, Gideon M Hirschfield MB BChir MRCP PhD
}

MV Zeman, GM Hirschfield. Autoantibodies and liver disease: Uses and abuses. Can J Gastroenterol 2010;24(4):225-231.

Confirming whether a patient has autoimmune liver disease is challenging, given its varied presentation and complex definitions. In the continued absence of pathognomonic serum markers, diagnosis requires evaluation of laboratory investigations and, frequently, a liver biopsy all of which need to be interpreted in the correct clinical context, with an emphasis on exclusion of viral infections, drug toxicity and metabolic disease. However, clear diagnosis is important for appropriate and timely therapy. Autoantibodies remain important tools for clinicians, and were the first proposed serological markers to aid in differentiating viral from chronic autoimmune hepatitis. Their presence is occasionally considered to be synonymous with autoimmune liver disease - a misinterpretation of their clinical significance. The present article summarizes the serum autoantibodies currently investigated in clinical and research practice, along with a description of their value in adult chronic liver diseases, with an emphasis on their appropriate use in the diagnosis and management of patients with autoimmune liver disease.

Key Words: Autoantibodies; Autoimmune hepatitis; Autoimmune liver disease; Primary biliary cirrhosis; Sclerosing cholangitis

\section{Auto-anticorps et maladie hépatique : Usages et abus}

Il est difficile de confirmer si un patient souffre de maladie hépatique autoimmune compte tenu de la variabilité des tableaux et de la complexité des définitions. En l'absence de marqueurs sériques pathognomoniques, le diagnostic repose sur l'examen des résultats d'analyses de laboratoire et souvent, sur une biopsie du foie, et tout doit être interprété dans le contexte clinique voulu, en se rappelant d'écarter les infections virales, les toxicités médicamenteuses et les maladies métaboliques. Il n'en reste pas moins que le diagnostic doit être clair pour qu'on puisse prodiguer un traitement approprié en temps voulu. Les auto-anticorps demeurent d'importants outils pour les cliniciens et ont été les premiers marqueurs sérologiques proposés pour aider à faire la distinction entre l'hépatite virale et l'hépatite auto-immune chronique. Leur présence est parfois considérée comme un synonyme de maladie hépatique auto-immune, ce qui est une interprétation erronée de leur portée clinique. Le présent article fait le point sur les auto-anticorps sériques actuellement testés en pratique clinique et en recherche et il décrit leur utilité dans les maladies hépatiques chroniques de l'adulte en rappelant leur emploi à bon escient pour le diagnostic et la prise en charge des patients souffrant de maladie du foie auto-immune.
A utoimmune liver disease comprises a number of chronic disorders of uncertain etiology characterized by immunemediated liver injury, frequently in the presence of circulating autoantibodies (Table 1) (1,2). Autoimmune hepatitis (AIH) (3) and primary biliary cirrhosis (PBC) (4), with their predominance in women and their association with other autoimmune diseases, are generally accepted to be autoimmune in origin, whereas there is greater debate regarding the predominantly large duct biliary diseases, primary sclerosing cholangitis (PSC) (5) and immunoglobulin (Ig) G4-associated autoimmune pancreatitis/sclerosing cholangitis (6).

Autoantibodies are immunoglobulins that recognize host antigens and may be present from birth without disease association (ie, natural autoantibodies) or occur later in life in response to antigenic stimulation (7). In disease, autoantibodies are considered pathological, although it remains unclear whether they are primary or secondary consequences of the underlying processes. There are considerably more autoantibodies than autoimmune diseases, and autoantibodies directed against the same broad antigenic target are not necessarily all identical. The mechanisms that lead to autoantibody production can differ between individuals with the same disease. While autoantibodies are often organ specific, autoimmunity is commonly tissue specific; furthermore, the autoantibodies themselves are usually not species specific. Therefore, conserved epitopes across species appear important. The presence of autoantibodies in healthy individuals is common; however, the presence of an autoantibody does not necessarily indicate the presence of an autoimmune disease, or its severity or response to therapy.

\section{HISTORICAL PERSPECTIVES AND BASICS OF AUTOANTIBODY TESTING}

Persistent liver injury and, in particular, 'chronic hepatitis' resulting in cirrhosis, became evident to clinicians in the latter half of the 20th century (8). Such liver disease was postulated to be the result of infection, alcohol intake, toxin exposure or nutritional disease. A variant of chronic hepatitis that affected mostly women and children was described. Appreciation of a potential autoimmune etiology emerged in the 1940s when Waldenström recognized the relevance of hypergammaglobulinemia and Kunkel described chronic liver disease in young, predominantly female patients with hypergammaglobulinemia (alongside extrahepatic symptoms including rash, arthralgias, fever and amenorrhea). Disease onset seemed insidious, with a course marked by episodic fevers and arthralgias, as well as a significant hepatic plasma cell infiltration. Zimmerman described a 36-year-old man with elevated serum gammaglobulins who died of subacute hepatic necrosis. It was believed that the initial liver injury was the result of injurious antiliver autoantibodies. 
TABLE 1

Autoantibodies commonly associated with chronic liver disease

\begin{tabular}{|c|c|c|}
\hline Autoantibody & Target & Notable association \\
\hline \multicolumn{3}{|l|}{ Nucleus } \\
\hline Antinuclear antibodies & Nuclear membranes and DNA (general) & Type 1 autoimmune hepatitis, primary biliary cirrhosis \\
\hline Histones & Nucleosomes & Type 1 autoimmune hepatitis, primary biliary cirrhosis \\
\hline $\begin{array}{l}\text { Perinuclear antineutrophil cytoplasmic } \\
\text { antibodies }\end{array}$ & Neutrophil granules & Type 1 autoimmune hepatitis, primary sclerosing cholangitis \\
\hline \multicolumn{3}{|l|}{ Microsomal } \\
\hline Liver kidney microsome-1 & Mitochondrial enzyme CYP450 2D6 & Type 2 autoimmune hepatitis \\
\hline \multicolumn{3}{|l|}{ Mitochondrial } \\
\hline Antimitochondrial antibodies & $\begin{array}{l}\text { ATPase-associated antigens of the inner } \\
\text { mitochondrial membrane }\end{array}$ & Primary biliary cirrhosis, autoimmune hepatitis \\
\hline \multicolumn{3}{|l|}{ Smooth muscle } \\
\hline Antismooth muscle antibodies & $\begin{array}{l}\text { Fibroblast actin, tubulin and intermediate } \\
\text { filaments (general) }\end{array}$ & $\begin{array}{l}\text { Type } 1 \text { autoimmune hepatitis, primary sclerosing cholangitis, } \\
\text { primary biliary cirrhosis }\end{array}$ \\
\hline Actin $^{*}$ & F-actin specifically & Type 1 autoimmune hepatitis \\
\hline \multicolumn{3}{|l|}{ Cytosol } \\
\hline Soluble liver and pancreas antigen* & $\begin{array}{l}\text { Glucuronyltransferase repressor transfer } \\
\text { RNA-associated protein }\end{array}$ & Autoimmune hepatitis \\
\hline Liver cytosol-1* & Formiminotransferase cyclodeaminase & Type 2 autoimmune hepatitis \\
\hline
\end{tabular}

${ }^{*}$ Cannot be measured outside the research setting

By the mid-1950s, blood tests to support an autoimmune etiology were emerging. The lupus erythematosus (LE) test was first used as a diagnostic test for systemic lupus erythematosus (SLE). The LE cell phenomenon involved incubating the peripheral blood of a patient suspected of having SLE with LE cell factor (found in the bone marrow of patients with SLE). During incubation, the LE cell factor reacted with damaged lymphocyte nuclei. Although recognized as useful, it soon became apparent that the test was neither sensitive nor specific for SLE. The factor responsible for the phenomenon is a family of antibodies to various nuclear antigens, broadly termed antinuclear antibodies (ANA), the major component of the LE cell factor being antihistone H1 (9).

It became apparent that patients with active chronic liver disease and hypergammaglobulinemia had LE cells, and it was noted that cortisone produced a dramatic improvement in symptoms. The liver disease was renamed lupoid hepatitis. The term AIH was first suggested in 1965 but was not endorsed until 1993.

Advances in the understanding of $\mathrm{PBC}$, first described in the 1850 s as liver disease associated with jaundice, splenomegaly and yellow skin plaques, also benefited from the newly emerging field of immunology. Investigation of jaundice traditionally involved surgical exploration to exclude gallstones and malignancy. The advent of immunofluorescence technology was pivotal to changing this. Its use identified a specific granular cytoplasmic fluorescence pattern on serum-stained sections of thyrotoxic thyroid gland and human gastric fundal mucosa in patients with presumed $\mathrm{PBC}$, whereas no fluorescence was detected in controls with chronic cholestasis. This serological test, later named antimitochondrial antibody (AMA), remains the most sensitive and specific marker for PBC.

Cell preparations, which include the human larynx epithelial cancer cell line (HEp-2) as well as cell preparations from rodent kidney and liver, are used for immunofluorescence-based assays. This reiterates the clinical paradox of disease specificity but serological cross-reactivity across species. Immunofluorescence patterns are subjective and most individuals screen positive if the serum concentration used is high enough. Strength of activity (ie, titre) can help clinicians determine the significance of the observation; however, regardless of titre, a positive test alone does not imply clinical significance (9). With the identification of the specific antigen reactivity of autoantibodies, a shift toward ELISA-based assays has occurred (10). Results or the antigenic epitopes tested, however, are not necessarily comparable between laboratories or assays.

\section{OVERVIEW OF COMMON AUTOANTIBODIES} ANA

ANA comprise antibodies with reactivity directed against nuclear membranes and DNA, although the target antigens (eg, centromeres, ribonucleoproteins and cyclin A [1]) are heterogeneous and incompletely defined. Classic AIH is associated with homogenous, speckled and nucleolar patterns, and in $\mathrm{AIH}$, the immunoflorescence pattern of ANA on HEp-2 cells is usually either homogenous (34\%) or speckled (38\%). The nuclear reactant in $\mathrm{AIH}$ is the same as that responsible for ANA reactivity in SLE - the nucleosome (chromatin). Other relevant patterns include anticentromere antibodies, multiple nuclear dot and antinuclear envelope antibodies (11). Perinuclear antineutrophil cytoplasmic antibodies (pANCA) are also antinuclear antibodies that react against the cytoplasmic components of neutrophils.

\section{Antismooth muscle antibodies}

Antismooth muscle antibodies (ASMA) are believed to be directed against either actin, tubulin or the intermediate filaments of the cell. Immunofluorescence assays using the HEp-2 cell line stain isolated fibroblasts and give the cell a characteristic 'cable' pattern. Antibodies to tubulin and intermediate filaments are commonly encountered in viral infections, including viral hepatitis and other autoimmune and nonautoimmune diseases. Given the lack of specificity, this has led some to suggest abandoning nonspecific ASMA testing in favour of more specific anti-F actin assays (12). 
TABLE 2

Clinical differences between the serological classifications of autoimmune hepatitis

\begin{tabular}{|c|c|c|}
\hline & \multicolumn{2}{|c|}{ Autoimmune hepatitis } \\
\hline & Type 1 & Type 2 \\
\hline Relative prevalence & $>80 \%$ & $20 \%$ in Europe, $4 \%$ in United States \\
\hline Autoantibodies commonly associated & Antinuclear, antismooth muscle & Liver kidney microsome-1 \\
\hline Patient demographic & Approximately $70 \%$ women & Female predominance \\
\hline Age of onset & $\begin{array}{l}\text { Peak incidence between } 16 \text { and } 30 \text { years of age, } \\
\text { although } 50 \% \text { are older than } 30 \text { years of age }\end{array}$ & $\begin{array}{l}\text { Average } 10 \text { years of age but seen in adults, specifically in } \\
\text { Europe }\end{array}$ \\
\hline $\begin{array}{l}\text { Other commonly associated autoimmune } \\
\text { diseases }\end{array}$ & $\begin{array}{l}\text { Prevalence of } 17 \% \text { to } 48 \% \text {. Thyroid disease, } \\
\text { synovitis, ulcerative colitis }\end{array}$ & $\begin{array}{l}\text { Prevalence unclear. Diabetes, thyroid disease, vitiligo, } \\
\text { pernicious anemia }\end{array}$ \\
\hline Presentation & Acute onset rare & $\begin{array}{l}\text { Frequently presents with cirrhosis in children and more } \\
\text { aggressively }\end{array}$ \\
\hline Response to treatment & Excellent & May be more treatment resistant \\
\hline Progression of disease & $\begin{array}{l}25 \% \text { have cirrhosis at diagnosis; } 45 \% \text { develop } \\
\text { cirrhosis }\end{array}$ & Approximately $80 \%$ develop cirrhosis \\
\hline
\end{tabular}

\section{Microsomal antibodies}

Antiliver-kidney microsome (LKM) was first described in individuals with AIH. 'Microsomal' is a misnomer because microsomes are the in vitro equivalent to particles of the endoplasmic reticulum (13). Different targeted autoantigens produce distinct immunofluoresence patterns that led to the subclassification from LKM to LKM-1 to LKM-4. LKM-1 (associated with type $2 \mathrm{AIH}$ ) reacts with the mitochondrial enzyme cytochrome P450 2D6 subtype (CYP2D6), inhibiting its activity in vivo. CYP2D6 metabolizes several known medications, including antihypertensives and benzodiazepines, and is genetically polymorphic. LKM-2 reacts with CYP450 2C9 and has been associated with the hepatitis caused by the medication tienilic acid, which was taken off the United States market in 1982. LKM-3, which has affinity for uridine diphosphate glucuronosyl transferase (14), has been associated with chronic hepatitis D and LKM-4, which recognizes CYP1A2 and CYP2A6 (with an immunofluorescence pattern indistinguishable from LKM-1), and has been described in patients with $\mathrm{AIH}$ associated with autoimmune polyendocrinopathy-candidiasis-ectodermal dystrophy. Practically, it is possible to misinterpret LKM-1 staining for AMA staining; thus, recourse to an ELISA-based LKM-1 assay may be required.

\section{AMA}

The antigenic target of AMA has been identified as a nonorganspecific, ATPase-associated antigen that is present on the inner mitochondrial membrane. It consists of several mitochondrial antigen/antibody systems numbered M1 to M9, of which only M2, M4, M8 and M9 are considered specific for PBC. The M2 subtype is most prevalent and is directed against related proteins of the alpha-keto acid dehydrogenase complex. The recognized major epitope is located on the E2 subunit of the pyruvate dehydrogenase complex. Additionally, AMA-M2 autoantibodies recognize the E1-alpha and E1-beta subunits of the same complex, and the E2 subunit of several other multienzyme complexes, such as the 2-oxo-glutarate dehydrogenase complex and the branchedchain 2-oxo acid dehydrogenase complex. AMA-negative PBC individuals have intense staining for the pyruvate dehydrogenase complex-E2 of the apical region of bile duct epithelial cells and equivalent $T$ cell specificities, demonstrating that even if the antibody is not detected, the biology is immunologically equivalent.
Although more than $90 \%$ of patients with PBC are AMApositive, AMA may be observed in nonautoimmune liver diseases such as SLE, rheumatoid arthritis and graft-versus-host disease. In addition, AMA (specifically AMA-MIT3, an assay capturing the three most prevalent antigens) can be found transiently in nearly one-half of all individuals with fulminant liver failure (15) and has been reported to develop transiently in a patient with AIH/PSC overlap syndrome (16).

\section{Autoantibodies in healthy individuals}

In asymptomatic blood donors, ANA prevalence (of any titre) has been found to vary between $4 \%$ and 26\%, with nearly $15 \%$ being positive at a 1:40 dilution. In addition to healthy controls, up to $10 \%$ of pregnant women, $30 \%$ of individuals with malignancy and up to $50 \%$ of individuals with infectious disease, are ANA positive. Up to $75 \%$ of ANA-positive individuals have no identifiable disease and long-term follow-up suggests a benign course in the majority $(17,18)$. ASMA can be present in up to $43 \%$ of normal healthy individuals, whereas AMA are estimated to be present in less than $1 \%$.

\section{CLINICAL INTERPRETATION OF AUTOANTIBODY RESULTS}

Autoantibody results must always be interpreted carefully because findings, although associated with autoimmune disease, do not generally relate to the severity, response to treatment or natural history.

\section{AIH}

Serological subtypes of AIH are commonly described (13) (Table 2) and the established scoring systems $(19,20)$ include ANA, ASMA and LKM autoantibodies as part of the evaluation. Eighty per cent of individuals with AIH have type $1 \mathrm{AIH}$, and ANA are usually present in titres greater than 1:160, although no specific ANA antigen has been identified. The speckled pattern has reportedly been found in younger patients with higher aminotransferase levels. Type 1 disease is also characterized by ASMA, which may be the only marker of type 1 $\mathrm{AIH}$ in children (1). Fifty per cent of individuals with type 1 AIH who are ASMA-positive have specific anti-F-actin antibodies. The use of anti-F-actin antibody is more predictive of $\mathrm{AIH}$, with a specificity and sensitivity of approximately $80 \%$ 
TABLE 3

Illustrative prevalences of antinuclear antibody (ANA) staining patterns in antimitochondrial (AMA)-positive/negative primary biliary cirrhosis (PBC)

\begin{tabular}{|c|c|c|c|c|}
\hline \multirow[b]{2}{*}{ Autoantibody } & \multicolumn{2}{|c|}{ Prevalence, \% } & \multicolumn{2}{|c|}{ PBC diagnosis, \% } \\
\hline & AMA-positive PBC & AMA-negative PBC & Specificity & Sensitivity \\
\hline ANA-positive* & $47-48$ & 68-100 & Very low & Very low \\
\hline Multiple nuclear dot-like* & $12-24$ & $38-41$ & Unknown & Unknown \\
\hline Speckled & 24 & $41-46$ & Unknown & Unknown \\
\hline Anticentromere & $14-20$ & $14-23$ & Unknown & Unknown \\
\hline
\end{tabular}

*Significant differences in the prevalence of ANA, multiple nuclear dot-like and perinuclear/rim-like membranous antibodies between AMA-positive and AMAnegative $P B C$. Data adapted from references 11 and 25

and $90 \%$, respectively (21); in $26 \%$ of individuals, it may be the sole marker of disease. ANA and ASMA levels fluctuate during the course of AIH and may disappear with corticosteroid therapy. Neither their titre at diagnosis nor their fluctuation during the course of illness predicts outcome.

The presence of LKM autoantibodies has been used to define type $2 \mathrm{AIH}$, which is typically ANA and ASMA negative. Type $2 \mathrm{AIH}$ tends to represent a disease more common in children and more aggressive than type $1 \mathrm{AIH}$. In Europe, as many as $20 \%$ of adults with AIH have type 2 AIH compared with only $4 \%$ in the United States. Whether type $2 \mathrm{AIH}$ is a distinct clinical disease from type 1 disease is unclear, although the presence of a definable antigen means that the disease is much better understood biologically (22).

A third phenotype of $\mathrm{AIH}$ (type $3 \mathrm{AIH}$ ) is occasionally referred to, and is serologically based on the presence of autoantibodies against soluble liver and pancreas antigen (SLA/LP). The two autoantibodies target the same antigen. Initially, individuals who were SLA/LP positive were classified as type 3 $\mathrm{AIH}$, because it was observed first among patients negative for ANA, ASMA and LKM autoantibodies. However, it has since been shown (1) that $74 \%$ of patients with so-called type $3 \mathrm{AIH}$ are also ANA and/or ASMA positive, and these individuals are clinically indistinct from those with type 1 disease; conversely, $10 \%$ to $30 \%$ of patients with type $1 \mathrm{AIH}$ are SLA/LP-positive when tested. Anti-LP is believed to be a better marker of AIH because normal individuals and those with nonhepatic disorders are invariably anti-LP negative. Approximately 33\% of individuals who are negative for all other conventional AIHrelated autoantibodies remain anti-LP positive.

pANCA has characteristically been associated with ulcerative colitis (UC), but $92 \%$ of individuals with $\mathrm{AIH}$, specifically type 1 , are also pANCA positive. Interestingly, the titres of pANCA are much higher in AIH than in PSC and the IgG subtypes differ. AIH can also be associated with doublestranded (ds) DNA antibodies, and some suggest that the overlap syndrome AIH/PBC may be characterized by anti-dsDNA positivity.

AMAs may be found in up to $20 \%$ of AIH individuals. They are usually lower in titre (1:40 or less) and, in some, represent false positives. The presence of AMA should not necessarily be interpreted as an $\mathrm{AIH} / \mathrm{PBC}$ overlap. One longterm study (23) of patients with AIH who were persistently AMA positive found those individuals to have the same laboratory, histological and clinical features, as well as the same treatment outcome compared with individuals who were AMA negative.

\section{PBC}

AMA are the hallmark of $\mathrm{PBC}$, and more than $90 \%$ of patients are positive for AMA staining by indirect immunofluorescence. Use of recombinant autoantigens and immunoblotting has increased the sensitivity and specificity of AMA testing to more than $95 \%$ (24). By adopting more specific testing, AMA have been measured in nearly $20 \%$ of individuals originally diagnosed as being AMA negative (25). AMA and, more specifically, AMA-M2, are such specific PBC autoantibodies that their presence is nearly diagnostic for PBC in the context of cholestasis without marked hepatitis (26). Their role in direct disease pathogenesis is less clear, although the AMA-specific $\mathrm{T}$ cell specificities of infiltrating lymphocytes in $\mathrm{PBC}$ suggest they are important. Histological evidence of $\mathrm{PBC}$ has been found in nearly $40 \%$ of asymptomatic individuals with normal liver enzymes who are AMA-positive, and long-term follow-up suggests PBC will develop in the majority. Although they may be stable in an individual for years, AMA titres can differ by a factor of more than 200 - with no clinical significance. The different type and number of mitochondrial antigens recognized in a specific patient are not associated with any features of disease, nor with its stage or prognosis. Although AMA titres may fall on treatment, they are not prognostic. Family members are not infrequently AMA positive, consistent with shared genetic and/or environmental factors.

AMA-negative $\mathrm{PBC}$ is now accepted to behave clinically in the same way as AMA-positive disease. If using the term, one needs to consider the assays used because true AMA-negative $\mathrm{PBC}$ requires exhaustive testing by immunofluorescence, immunoblotting and ELISA.

One-half of patients with $\mathrm{PBC}$ are also ANA positive (Table 3) and, unlike the nonspecific ANA in AIH, highly PBC-specific ANA target antigens have been identified. ANA immunofluorescence is described either as a perinuclear/rim-like membranous pattern formed by antiglycoprotein (anti-gp) 210 and lamin B receptor (constituents of the nuclear envelope), a multiple nuclear dot-like pattern formed by anti-Sp100 and promyelocytic leukemia protein (two autoantibodies that colocalize), or a centromere pattern.

Immunoassays testing for the presence of anti-gp210 and antinucleoporin $62 \mathrm{KDa}$, a pore complex that anchors to the nuclear membrane in the presence of anti-gp210, suggest that 
these antibodies may be found in $10 \%$ to $47 \%$, and $32 \%$ of patients with $\mathrm{PBC}$, respectively. In addition, anti-Sp100 antibodies may be found in $18 \%$ to $44 \%$ of individuals with PBC. Multiple nuclear dot and perinuclear/rim-like membranous patterns are extremely specific for PBC. They are so specific that these ANA patterns can be used as positive PBC markers in individuals who are AMA negative, with ANA being more commonly found in AMA-negative PBC. Oertelt et al (25) reported $100 \%$ of patients to be ANA positive, although others (11) suggest a lower, but still significant proportion.

Anti-gp210 is associated with aggressive disease and progression to liver failure in individuals with PBC. Individuals whose anti-gp210 titres changed from positive to negative during ursodeoxycholic acid therapy were found to show no progression to liver failure (27). As with AMA, anti-gp210 titres have been found to persist after liver transplantation but the clinical significance is unknown. In addition, the anticentromere ANA pattern has been associated with the development of portal hypertension but not hepatic failure (27). Unlike anti-gp210, anticentromere antibodies are detected before the onset of $\mathrm{PBC}$, and titres do not change during the course of the disease.

\section{Sclerosing cholangitis}

Sclerosing cholangitis, when evident radiographically by either endoscopic retrograde cholangiopancreatography or magnetic resonance cholangiopancreatography, is defined as 'large duct' disease, whereas 'small duct' disease refers to duct fibrosis and duct loss seen only on biopsy. In either case, the definition of 'primary' assumes secondary causes, including IgG4-related disease, have been ruled out. Several autoantibodies have been found in individuals with PSC, although none are disease specific, and specificities and prevalence in PSC varies among studies.

The most studied autoantibody in patients with PSC is pANCA, an ANA subtype, as described previously. The general prevalence of ANCA in PSC can be up to 93\%, with the pANCA subtype found in up to $94 \%$ of individuals. In a review of 19 different studies (28), the mean prevalence of pANCA in PSC was calculated to be $63 \%$. However, pANCA is nonspecific and can be found in nearly equal rates in AIH and PBC, and even in $5 \%$ of healthy control patients. Its sensitivity and specificity varies among studies, and is estimated to be as high as $61 \%$ and $78 \%$, respectively, at a cut-off of $1: 40$. Testing is, therefore, not of any immediate clinical utility, although a report suggested it may be relevant to small duct disease.

ANA and ASMA have been detected in PSC, but as with $\mathrm{AIH}$, prevalence varies among studies. ANA were reported in $8 \%$ to $77 \%$ of patients with PSC, and ASMA in $0 \%$ to $83 \%$ (28).

The most closely associated disease-specific antibodies for PSC are those directed against biliary cells, suggesting not only an immune-mediated response but also that antigens expressed in the biliary epithelium may induce self-reactivity. Antibodies to human tropomyosin isoform, a protein expressed in both colonic and biliary epithelium (29), has been found in individuals with PSC but without UC, with a prevalence of $100 \%$ (eight of eight) versus in 69\% (33 of 48) of control UC patients (30). A second antibody, antibiliary epithelial cell, has been documented in PSC and PBC patients, with a prevalence of $63 \%$ and $37 \%$, respectively (31).

\section{Hepatitis C}

\section{MISCELLANEOUS SCENARIOS}

ANA, ASMA and LKM have all been described in hepatitis C (HCV), and while AIH is possible coincidentally, their mere presence is not indicative per se. ANA are found in approximately $5 \%$ of individuals with $\mathrm{HCV}$ at titres of more than 1:100 (21). HCV, however, can be distinguished from type 1 $\mathrm{AIH}$ by virtue of the fact that classical HCV does not display prominent plasma cell infiltrate on liver biopsy and, typically, $\mathrm{HCV}$ is not associated with marked elevations of serum IgG unless in the setting of cirrhosis. ASMA are seen in HCV, although the mean titre is only 1:40 versus 1:320 in type 1 AIH (21). AMA are infrequently described in individuals with HCV.

LKM was recognized as an antibody prevalent in HCV disease. Although found in $50 \%$ to $86 \%$ of German and Italian patients with HCV, LKM has only been found in $1 \%$ of Western European and American patients with HCV infection. There is homology between the CYP450 2D6 and the HCV viral genome, possibly suggesting molecular mimicry. Patients with LKM-1 who are infected with $\mathrm{HCV}$ react less frequently to recombinant CYP2D6 than do patients who are HCV negative, and LKM-1 antibody specificities in AIH have been shown to be different to those in HCV.

\section{Celiac disease}

Celiac disease has an estimated prevalence of approximately $1 \%$ in North America. It can occur coincidentally with liver disease, or may itself be a cause of liver test abnormalities and liver dysfunction, with a prevalence of celiac disease in those with cryptogenic cirrhosis estimated to be $4 \%$. However, celiac disease has been reported in approximately $6 \%$ of individuals with $\mathrm{AIH}$ and in $3 \%$ with PSC. The prevalence of $\mathrm{PBC}$ in celiac disease is approximately $3 \%$, whereas the prevalence of histological celiac disease in PBC is approximately $6 \%$. The false-positive rate of antitransglutaminase antibody testing has, however, been estimated to be nearly 50\% in those with PBC and AIH, and depends on the assay used. Therefore, in testing for celiac disease in patients with autoimmune liver disease, a small bowel biopsy must follow a positive serological result.

\section{Postliver transplant}

Autoimmune hepatitis can occur de novo after liver transplant or recur in individuals transplanted because of $\mathrm{AIH}$. Recurrences of $\mathrm{AIH}$, the development of an alloimmune phenomenon or allograft dysfunction mimicking autoimmune hepatitis, are difficult to differentiate based on histological findings. In addition, alloimmune hepatitis does not differ from the recurrence of classic AIH based on serology. In both patients, it can present with elevated IgG, ANA and ASMA levels. Alloimmune hepatitis has, however, been associated with a mismatch between the expression of glutathione S-transferase T1 (GSTT1), a metabolizing enzyme, in the recipient versus the donor (null recipient/positive donor) and is marked by the presence of serum anti-GSTT1 antibodies. PBC may recur in the liver allograft. While AMA titres usually decline post-transplant, they remain detectable by sensitive assays. Graft PBC cannot be diagnosed on the basis of liver enzyme profile and AMA presence because of the wide differential; histological evaluation is essential. 


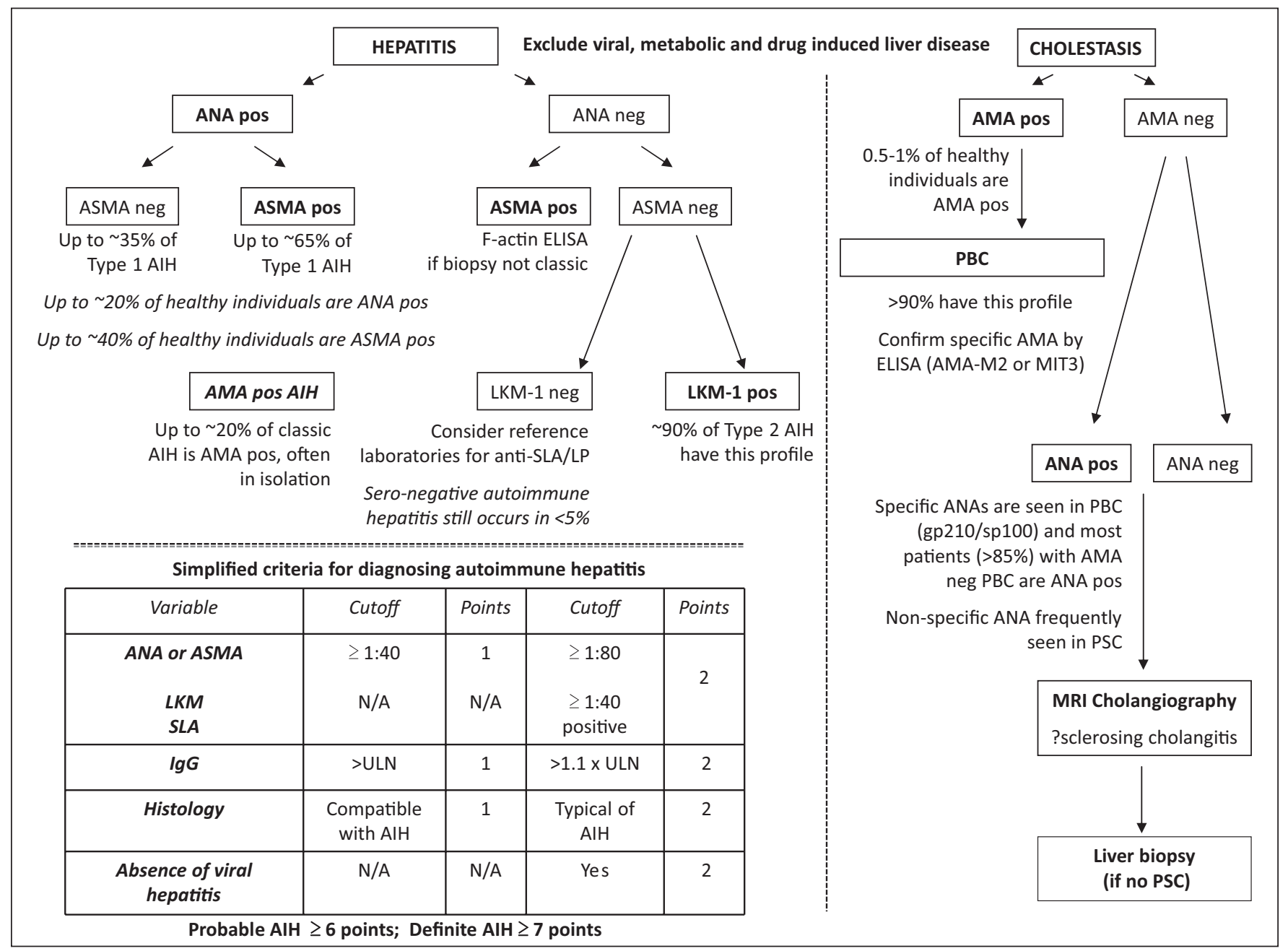

Figure 1) Guide to practical interpretation of autoantibodies in the diagnosis of autoimmune liver disease. Simplified flow chart for interpretation of autoantibodies in the differential diagnosis of hepatitis and cholestasis, alongside the simplified criteria for diagnosing autoimmune hepatitis (AIH) (20). AMA Antimitochondrial antibody; ANA Antinuclear antibodies; ASMA Antismooth muscle antibodies; gp Glycoprotein; Ig Immunoglobulin; LKM Liver kidney microsome; MIT Mitochondrial; MRI Magnetic resonance imaging; neg Negative; N/A Not applicable; PBC Primary biliary cirrhosis; pos Positive; PSC Primary sclerosing cholangitis; SLA Soluble liver antigen; SLA/LP Soluble liver and pancreas antigen; ULN Upper limit of normal. Data adapted from references 13 and 32

\section{PRACTICAL ADVICE}

A suggested diagnostic algorithm is presented in Figure 1 $(13,32)$. Patient selection is the most important factor to consider when interpreting a positive autoantibody result, because the positive predictive value of a test is based on the patient population. For example, the positive predictive value of a positive ANA test in an individual with elevated liver transaminase levels is greater than in a healthy individual with normal liver enzymes. ANA can be positive in up to $20 \%$ to $30 \%$ of individuals, depending on the assay used. However, up to $65 \%$ of individuals with type $1 \mathrm{AIH}$ are also ANA positive (35\% are solely ANA positive, but $65 \%$ are ANA and ASMA positive). Because only one in 100,000 individuals have type 1 $\mathrm{AIH}$, false-positives predominate even if individuals are ANA and/or ASMA positive (99.98\% false-positive rate), with a true positive rate of only three in 100,000 . Given the low pretest probability of detecting an autoimmune liver disease by autoantibody testing alone, using these investigations in healthy individuals is neither useful nor productive, and even in patients with symptoms/laboratory abnormalities, there are clear difficulties.

When an autoimmune liver disease is suspected based on clinical, biochemical or histological pattern, autoantibody testing of ANA, ASMA, LKM-1 and AMA can clearly help with the diagnosis. Titres can vary throughout the course of the disease and, therefore, a negative test or a low titre should not exclude a diagnosis; repeat testing may be appropriate during the initial workup. In adults, a titre of 1:40 or greater is considered positive for pathology, whereas in children, a cut-off of 1:20 or greater for ANA and ASMA, and 1:10 or greater for LKM-1 are considered positive (10).

\section{CONCLUSION}

Autoantibody testing has contributed to both biological and clinical insights in managing patients with liver disease, particularly that which is presumed to be autoimmune in nature. To date, testing is imprecise and future serological markers of disease are likely to be based on much more specific antigen-based 
assays. Clinicians need to appreciate the value and limitation of testing (33). Developments from proteomic studies, in particular, are anticipated to ultimately provide the more specific markers clinicians are looking for (34).

\section{REFERENCES}

1. Strassburg CP, Manns MP. Autoantibodies and autoantigens in autoimmune hepatitis. Semin Liver Dis 2002;22:339-52.

2. Manns MP, Vogel A. Autoimmune hepatitis, from mechanisms to therapy. Hepatology 2006;43:S132-44.

3. Czaja AJ, Freese DK. Diagnosis and treatment of autoimmune hepatitis. Hepatology 2002;36:479-97.

4. Heathcote EJ. Management of primary biliary cirrhosis. The American Association for the Study of Liver Diseases practice guidelines. Hepatology 2000;31:1005-13.

5. LaRusso NF, Shneider BL, Black D, et al. Primary sclerosing cholangitis: Summary of a workshop. Hepatology 2006;44:746-64.

6. Montano-Loza AJ, Lalor E, Mason AL. Recognizing immunoglobulin G4-related overlap syndromes in patients with pancreatic and hepatobiliary diseases. Can J Gastroenterol 2008;22:840-6.

7. Elkon K, Casali P. Nature and functions of autoantibodies. Nat Clin Pract Rheumatol 2008;4:491-8.

8. Mackay IR. Historical reflections on autoimmune hepatitis. World J Gastroenterol 2008;14:3292-300.

9. Keren DF. Antinuclear antibody testing. Clin Lab Med 2002;22:447-74.

10. Vergani D, Alvarez F, Bianchi FB, et al. Liver autoimmune serology: A consensus statement from the committee for autoimmune serology of the International Autoimmune Hepatitis Group. J Hepatol 2004;41:677-83.

11. Muratori L, Granito A, Muratori P, et al. Antimitochondrial antibodies and other antibodies in primary biliary cirrhosis: Diagnostic and prognostic value. Clin Liver Dis 2008;12:261-76.

12. Frenzel C, Herkel J, Luth S, et al. Evaluation of F-actin ELISA for the diagnosis of autoimmune hepatitis. Am J Gastroenterol 2006;101:2731-6.

13. Bogdanos DP, Invernizzi P, Mackay IR, Vergani D. Autoimmune liver serology: Current diagnostic and clinical challenges. World J Gastroenterol 2008;14:3374-87.

14. Philipp T, Durazzo M, Trautwein C, et al. Recognition of uridine diphosphate glucuronosyl transferases by LKM-3 antibodies in chronic hepatitis D. Lancet 1994;344:578-81.

15. Leung PS, Rossaro L, Davis PA, et al. Antimitochondrial antibodies in acute liver failure: Implications for primary biliary cirrhosis. Hepatology 2007;46:1436-42.

16. Bhat M, Guindi M, Heathcote EJ, Hirschfield GM. Transient development of anti-mitochondrial antibodies accompanies autoimmune hepatitis-sclerosing cholangitis overlap. Gut 2009;58:152-3.

17. Wijeyesinghe U, Russell AS. Outcome of high titer antinuclear antibody positivity in individuals without connective tissue disease: A 10-year follow-up. Clin Rheumatol 2008;27:1399-402.

18. Kavanaugh A, Tomar R, Reveille J, et al. Guidelines for clinical use of the antinuclear antibody test and tests for specific autoantibodies to nuclear antigens. American College of Pathologists. Arch Pathol Lab Med 2000;124:71-81.

19. Alvarez F, Berg PA, Bianchi FB, et al. International Autoimmune Hepatitis Group Report: Review of criteria for diagnosis of autoimmune hepatitis. J Hepatol 1999;31:929-38.

20. Hennes EM, Zeniya M, Czaja AJ, et al. Simplified criteria for the diagnosis of autoimmune hepatitis. Hepatology 2008;48:169-76.

21. Cassani F, Cataleta M, Valentini P, et al. Serum autoantibodies in chronic hepatitis C: Comparison with autoimmune hepatitis and impact on the disease profile. Hepatology 1997;26:561-6.

22. Holdener M, Hintermann E, Bayer M, et al. Breaking tolerance to the natural human liver autoantigen cytochrome P450 2D6 by virus infection. J Exp Med 2008;205:1409-22.

23. O'Brien C, Joshi S, Feld JJ, et al. Long-term follow-up of antimitochondrial antibody-positive autoimmune hepatitis. Hepatology 2008;48:550-6.

24. Kaplan MM, Gershwin ME. Primary biliary cirrhosis. N Engl J Med 2005;353:1261-73.

25. Oertelt S, Rieger R, Selmi C, et al. A sensitive bead assay for antimitochondrial antibodies: Chipping away at AMA-negative primary biliary cirrhosis. Hepatology 2007;45:659-65.

26. Zein CO, Angulo P, Lindor KD. When is liver biopsy needed in the diagnosis of primary biliary cirrhosis? Clin Gastroenterol Hepatol 2003;1:89-95.

27. Nakamura M, Kondo H, Mori T, et al. Anti-gp210 and anticentromere antibodies are different risk factors for the progression of primary biliary cirrhosis. Hepatology 2007;45:118-27.

28. Hov JR, Boberg KM, Karlsen TH. Autoantibodies in primary sclerosing cholangitis. World J Gastroenterol 2008;14:3781-91.

29. Das KM. Immunopathogenesis of primary sclerosing cholangitis: Possible role of a shared colonic and biliary epithelial antigen. J Gastrol Hepatol 2004;19:S290-4.

30. Sakamaki S, Takayanagi N, Yoshizaki N, et al. Autoantibodies against the specific epitope of human tropomyosin(s) detected by a peptide based enzyme immunoassay in sera of patients with ulcerative colitis show antibody dependent cell mediated cytotoxicity against HLA-DPw9 transfected L cells. Gut 2000;47:236-41.

31. Xu B, Broome U, Ericzon BG, Sumitran-Holgersson S. High frequency of autoantibodies in patients with primary sclerosing cholangitis that bind biliary epithelial cells and induce expression of CD44 and production of interleukin 6. Gut 2002;51:120-7.

32. Rigopoulou EI, Mytilinaiou M, Romanidou O, et al. Autoimmune hepatitis-specific antibodies against soluble liver antigen and liver cytosol type 1 in patients with chronic viral hepatitis. J Autoimmune Dis 2007;4:2.

33. Fritzler MJ. Challenges to the use of autoantibodies as predictors of disease onset, diagnosis and outcomes. Autoimmun Rev 2008;7:616-20.

34. Tahiri F, Le Naour F, Huguet $S$, et al. Identification of plasma membrane autoantigens in autoimmune hepatitis type 1 using a proteomics tool. Hepatology 2008;47:937-48. 


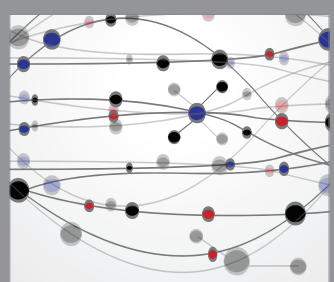

The Scientific World Journal
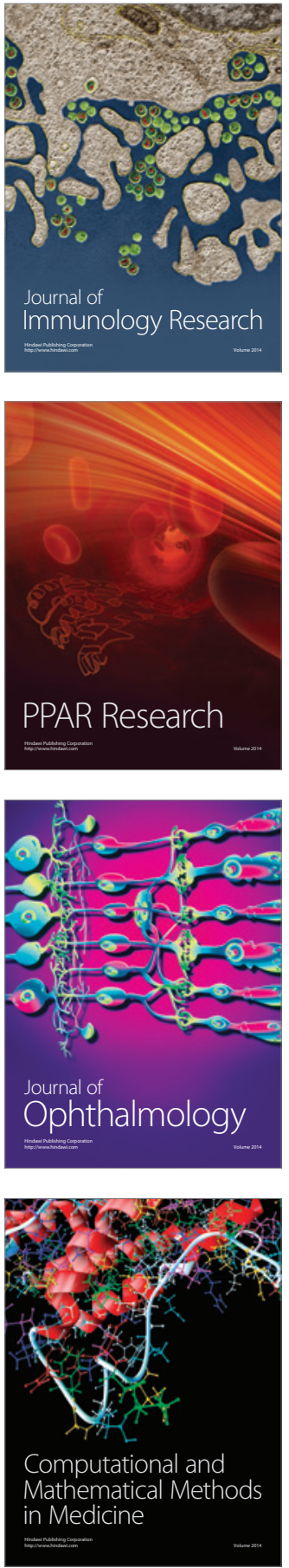

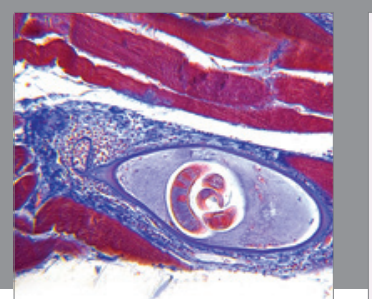

Gastroenterology Research and Practice

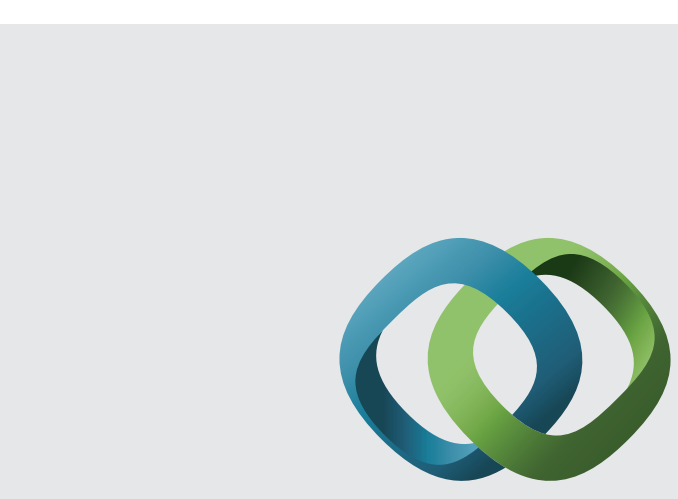

\section{Hindawi}

Submit your manuscripts at

http://www.hindawi.com
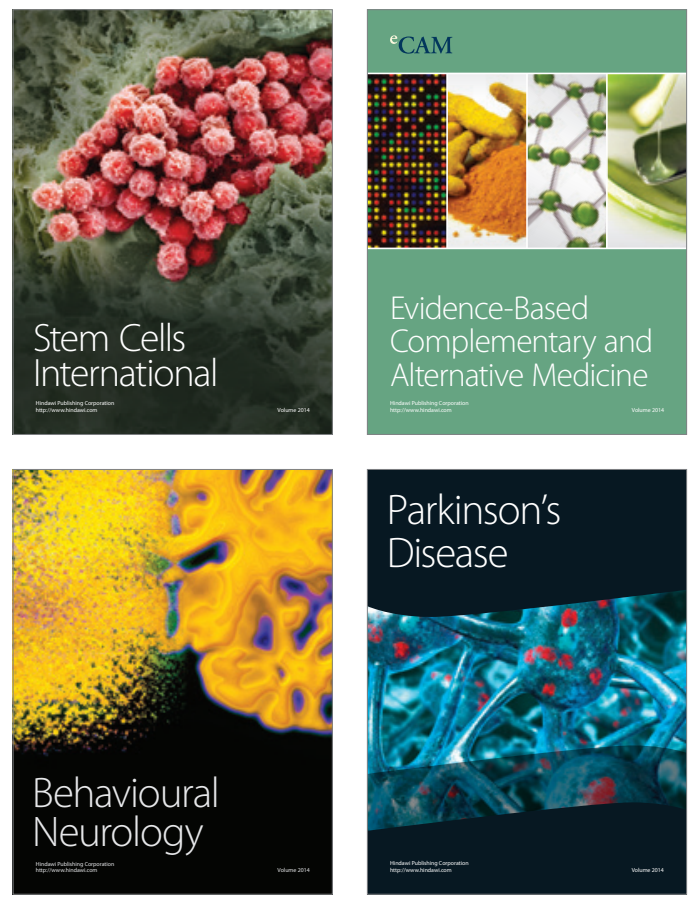
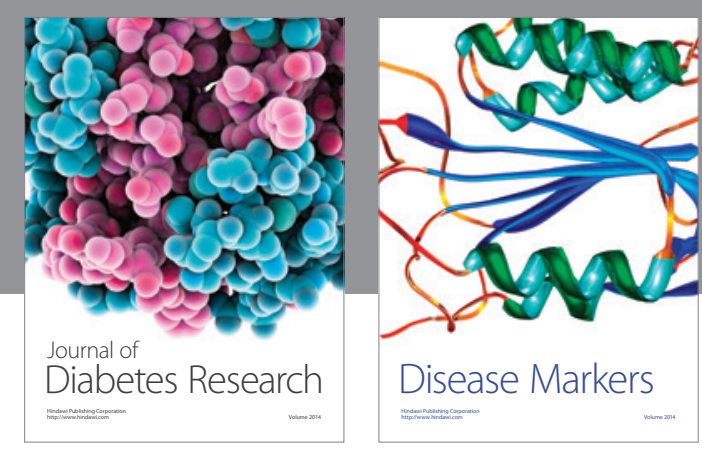

Disease Markers
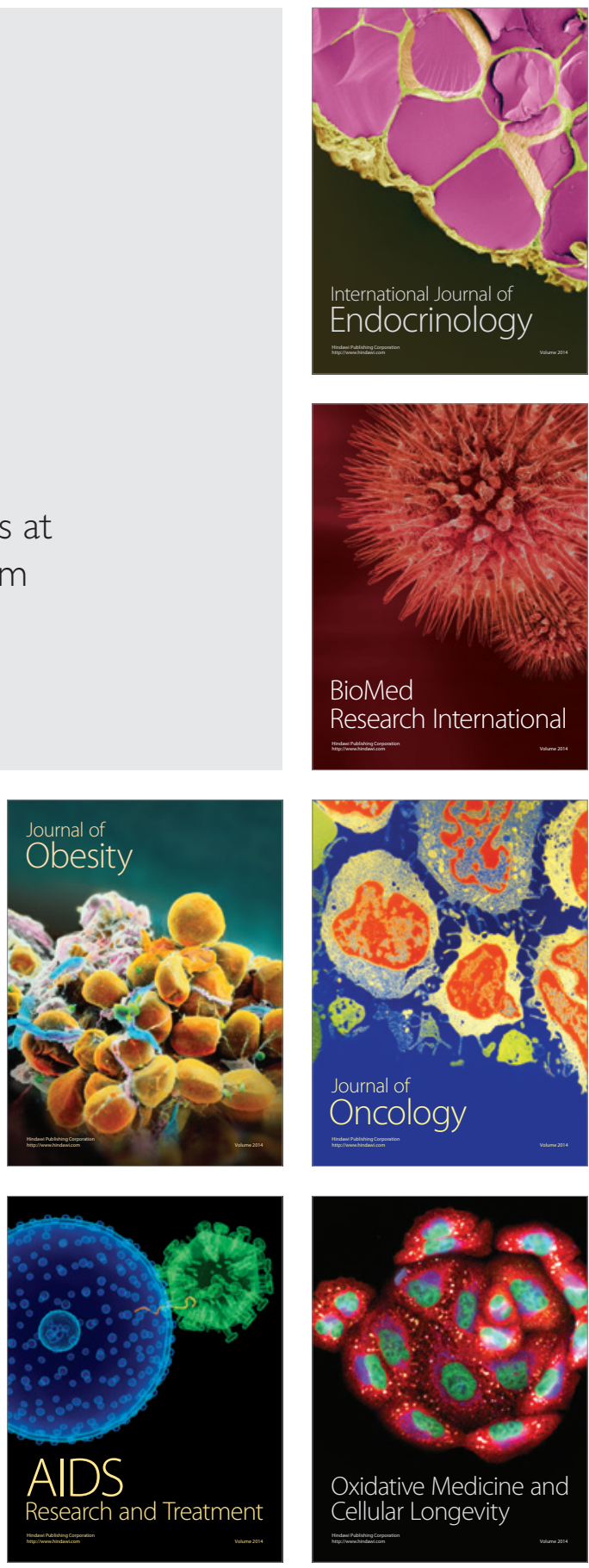